\title{
Considerações sobre eficiência administrativa relacionado aos exames de ultra-sonografia, tomografia computadorizada e ressonância magnética nas afecções orbitárias
}

\author{
Reasons for the administrative efficiency related to \\ ultrassonography, computed tomography and magnetic \\ resonance imaging tests in orbital diseases
}

Magno Watanabe $\uparrow$, Carlos Augusto de Morais ${ }^{2}$, Abelardo de Souza Couto Júnior

\section{Resumo}

Objetivos: Enfocar os exames de ultra-sonografia (US), tomografia computadorizada (TC) e ressonância magnética (RM), levantando, revisando e discutindo as seguintes medidas na melhoria da eficiência destes procedimentos na órbita: 1-Defasagem dos honorários médicos; 2-Vantagens e desvantagens dos exames;3-Diretrizes clínicas. Métodos: No período de julho de 2002 a março de 2003, realizou-se um levantamento dos valores dos exames orbitários de US, TC e RM pagos pelo Sistema Único de Saúde - SUS, operadoras de planos e seguros de saúde e particulares cobrados em três clínicas oftalmológicas e três clínicas radiológicas referenciadas. Pesquisa bibliográfica e revisão na literatura dos referidos exames e suas diretrizes clínicas nas afeccões orbitárias. Resultados: 1-Valores dos exames em reais (R\$): US: 9,05 - SUS / 81,77 - AMB 90/ 57,01 - AMB/CIEFAS 93/102,00 - AMB/LPM 99 / 158,33 - particular. TC: 86,76 - SUS / 193,70 - AMB 90 / 196,30 - AMB/CIEFAS 93 / 204,12 - AMB/LPM 99 / 255,33 - particular. RM: 268,75 - SUS / 475,80 - AMB 90 e AMB/ CIEFAS 93/472,50 - AMB/LPM 99/563,33 - particular;2-Vantagens e desvantagens dos exames descritos de acordo com a revisão da literatura; 3 - As doenças da órbita não têm ainda listadas suas diretrizes. Conclusão: Estes exames são os mais utilizados e consagrados pela sua eficácia no estudo da órbita. Para uma maior eficiência, devemos considerar as seguintes recomendações administrativas: 1- Modernização e atualização das tabelas de honorários com índices mínimos e mais justos na remuneração dos exames com reembolso dos insumos utilizados como filmes radiológicos e contrastes: 2- Promover e estimular a educação médica continuada, evitando o excesso de exames desnecessários ou inapropriados: 3-Elaboração e implementação de diretrizes e protocolos clínicos baseados em evidências nas principais doenças orbitárias que possam ser utilizados como referência.

Descritores: Administração da prática médica; Ultra-sonografia; Tomografia computadorizada por raios X; Imagem por ressonância magnética; Órbita

\footnotetext{
${ }^{1}$ Mestre em Administração da Prática Oftalmológica pela Universidade Federal de São Paulo - UNIFESP - São Paulo (SP), Brasil; Chefe do Setor de Ultra-sonografia do Instituto Benjamin Constant - IBC - Rio de Janeiro (RJ), Brasil; (In memoriam)

${ }^{2}$ Residente de Oftalmologia do Instituto Benjamin Constant - IBC - Rio de Janeiro (RJ), Brasil:

${ }^{3}$ Doutor, Professor Adjunto de Oftalmologia da Faculdade de Medicina de Valença - Rio de Janeiro (RJ) - Brasil; Coordenador da Residência Médica em Oftalmologia e Chefe do Setor de Plástica Ocular e Órbita do Instituto Benjamin Constant - IBC - Rio de Janeiro (RJ) - Brasil; Professor da Pós-graduação em Oftalmologia da Pontifícia Universidade Católica - PUC - Rio de Janeiro (RJ) - Brasil
} 


\section{INTRODUÇÃO}

D esde a descoberta dos raios X por Roëntgen em 1895, novas modalidades propedêuticas de imagem trouxeram importantes conquistas na detecção, localização e diferenciação de diversas patologias, contribuindo assim na prevenção, diagnóstico, prognóstico e tratamento das doenças ${ }^{(1)}$.

Entre as principais afecções que podem acometer a órbita estão a orbitopatia distireoidiana, os processos inflamatórios e infecciosos, os tumores, os traumatismos e os corpos estranhos ${ }^{(2)}$. Dependendo da sua gravidade, tais lesões podem levar a defeitos estéticos, perda visual e outras seqüelas importantes ${ }^{(3)}$.

O estudo da órbita é uma área multidisciplinar de difícil avaliação e conduta. Geralmente, envolve além da oftalmologia, a interação com outras especialidades (como a radiologia, a otorrinolaringologia, a endocrinologia, a neurocirurgia, a patologia, a oncologia e a medicina interna) $)^{(3)}$, necessitando muitas vezes exames diagnósticos de imagem, alguns de última geração e de alto custo como a ressonância magnética (RM) e, recentemente, a tomografia por emissão de pósitrons (TEP). Outros exames incluem a radiografia simples ( $\mathrm{Rx}$ simples), a ultra-sonografia (US), a tomografia computadorizada (TC), a angiografia e o doppler colorido ${ }^{(4-8)}$. Atualmente, os mais utilizados são a US, a TC e a RM ${ }^{(2,8-9)}$.

Trabalhos científicos comprovam a acurácia e a eficácia destes exames, com excelentes resultados em relação as suas aplicações clínicas. Entretanto, avaliações econômicas sobre a eficiência destes procedimentos têm sido pouco pesquisados ${ }^{(10-11)}$. A eficiência é um importante conceito administrativo atual que engloba a eficácia de determinado procedimento ou medida, dentro das condições reais e o custo dos recursos empregados, visando melhorar a qualidade e o custo-efetividade da saúde ${ }^{(5 \cdot)}$.

Apesar dos constantes avanços tecnológicos na área de imagem, três fatores vêm contribuindo para a ineficiência na utilização destes exames com conseqüente aumento dos gastos para as instituições de saúde públicas e privadas e poucos benefícios para os pacientes:

1- Os altos custos em detrimento com a baixa remuneração dos procedimentos propiciando a auto-geração de exames ${ }^{(12)}$;

2- O desconhecimento médico ou a confiança exagerada nos mesmos, levando a iatrogenias e erros diagnósticos ${ }^{(5,13-14)}$.

3- Ausência de diretrizes ou protocolos clínicos nas afecções orbitárias ${ }^{(15-16)}$.

\section{Овjetivos}

Este estudo enfoca os exames de US, TC e RM, procurando levantar, revisar e discutir as seguintes medidas na melhoria da eficiência relacionados a estes procedimentos na órbita:

1- Defasagem dos honorários médicos;

2- Atualização médica (vantagens e desvantagens dos exames);

3- Diretrizes clínicas nas doenças orbitárias.

\section{Métodos}

No período de julho de 2002 a março de 2003, foi realizado um levantamento dos valores dos exames orbitários de US, TC e RM pagos pelo Sistema Único de Saúde - SUS, operadoras de planos e seguros de saúde e particulares cobrados em três clínicas oftalmológicas e três clínicas radiológicas referenciadas, localizadas na zona sul da cidade do Rio de Janeiro. Pesquisa bibliográfica e revisão na literatura dos referidos exames e suas diretrizes clínicas nas afecções orbitárias.

\section{Resultados}

\section{Honorários médicos}

As operadoras de planos e seguros de saúde pesquisadas remuneram os exames orbitários de US, TC e RM principalmente pelas Tabelas de Honorários Médicos (THM) da Associação Médica Brasileira - AMB 1990 e do Comitê de Integração de Entidades Fechadas de Assistência à Saúde - CIEFAS/AMB 1993. Nestas tabelas, o fator de correção e conversão adotado foi o coeficiente de honorários $(\mathrm{CH})$. O fator de $\mathrm{CH}$ pode variar, dependendo da empresa contratante de serviços. No Quadro 1 estão listadas as diversas empresas, suas respectivas tabelas adotadas e o fator de $\mathrm{CH}$ utilizados.

Os valores dos procedimentos fixados em $\mathrm{CH}$, pagos pelas tabelas AMB 90 e AMB/CIEFAS 93, encontram-se na Tabela 1. Para o exame de US de órbita foi calculado o valor do exame bilateral, resultado da soma de $100 \%$ do exame de uma órbita $+70 \%$ da órbita contralateral (Tabelas 1 e 3).

$\mathrm{Na}$ Tabela 2 encontram-se os valores dos exames particulares. Nos procedimentos de TC e RM, neste levantamento, não está incluso o valor do contraste endovenoso (iônico ou não iônico e paramagnético).

Os serviços credenciados pelo SUS, tanto públicos quanto privados, recebem sua remuneração para os exames estudados pela tabela de procedimentos do Sistema de Informações Ambulatoriais do Sistema Único de Saúde - SIA/SUS. 


\section{Quadro 1}

Tabela utilizada e fator de coeficiente de honorários (CH) segundo as principais instituições privadas (operadoras de planos e seguros de saúde) pagadoras de exames de imagem da órbita na cidade do Rio de Janeiro, 2002/2003

\begin{tabular}{lcc}
\hline Instituição & Tabela & Fator (CH) \\
\hline Amil & AMB 90 & 0.27 \\
Assefaz & CIEFAS 93 & 0.24 \\
CAARJ & CIEFAS 93 & 0.25 \\
Caberj & CIEFAS 93 & 0.24 \\
CASSI & CIEFAS 93 & 0.24 \\
DIX & AMB 90 & 0.24 \\
EMBRATEL & CIEFAS 93 & 0.24 \\
Golden Cross & AMB 90 & 0.28 \\
Medial & AMB 90 & 0.28 \\
Medservice & AMB 90 & 0.27 \\
Petrobras & CIEFAS 93 & 0.24 \\
Saúde Bradesco & AMB 90 & 0.28 \\
Sul América & AMB 90 & 0.27 \\
UNIMED & CIEFAS 93 & 0.27 (pessoa física) \\
UNIMED & AMB 90 & 0.24 (pessoa jurídica) \\
\hline
\end{tabular}

Legenda:

AMB - Associação Médica Brasileira

CIEFAS - Comitê de Integração de Entidades Fechadas de Assistência à Saúde Fontes: Clínicas particulares ofttalmológicas e radiológicas na cidade do Rio de Janeiro

Na Tabela 3, encontram-se os valores pagos peloSUS, operadoras de planos e seguros de saúde e particulares.

Nos exames de US, o valor pago inclui a utilização do aparelho, a remuneração do ultra-sonografista e todos os materiais utilizados como: colírio anestésico, gel ou metilcelulose e papel térmico de impressão (Tabelas 1,2 e 3).

A remuneração dos exames de TC e RM de acordo com as tabelas AMB 90, AMB/CIEFAS 93 e Lista de Procedimentos Médicos - AMB/LPM 99, compreendem os valores dos custos operacionais e honorários médicos. Filmes radiológicos, contrastes, medicamentos, guias, catéteres e demais insumos são cobrados à parte, e não foram levantados nesta pesquisa (Tabelas 1 e 3 ).

Nos procedimentos de TCe RM pagos peloSUS, não estão previstos reembolsos de filmes radiológicos, insumos ou contrastes. Portanto, a remuneração abrange o custo total do exame. O valor do procedimento de US de órbita é único, tanto para o exame uni ou bilateral (Tabela 3 ).

\section{Vantagens e desvantagens dos exames}

As vantagens e desvantagens dos procedimentos orbitários de imagem foram pesquisadas nos exames de US (Quadro 2), TC (Quadro 3) e RM (Quadro 4).

\section{Diretrizes clínicas}

Em outubro de 2001, o Conselho Federal de Medicina-CFM e a AMB, utilizando os princípios da medi-
Tabela 1

Valores dos procedimentos orbitários de US, TC e RM em coeficientes de honorários $(\mathrm{CH})$ de acordo com as instituições privadas (TABELAS AMB 1990 e AMB/CIEFAS 1993) - cidade do Rio de Janeiro, 2002/ 2003

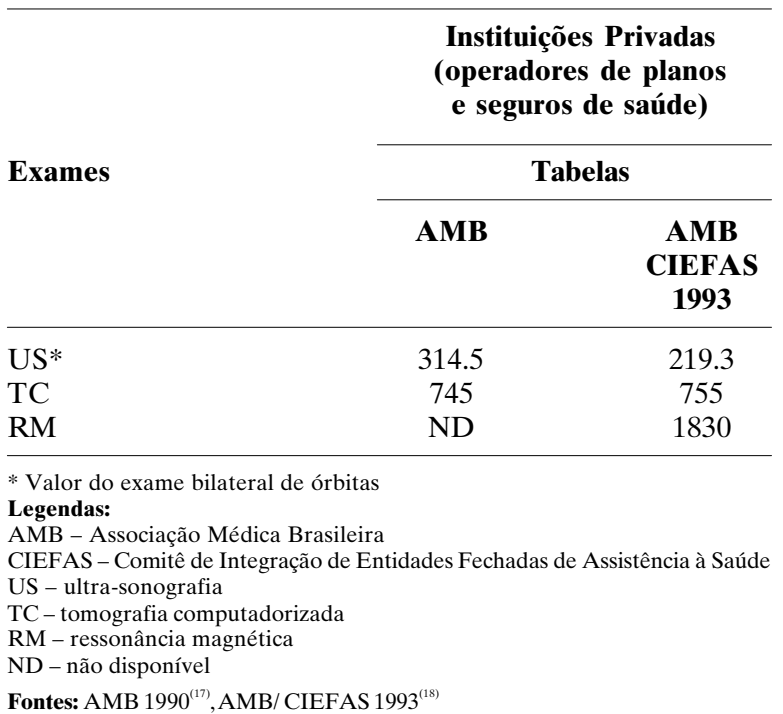

Tabela 2

Valores dos exames orbitários de US, TC e RM em reais (R\$) em Clínicas particulares na cidade do Rio de Janeiro, 2002/ 2003

\begin{tabular}{lcccc}
\hline & \multicolumn{4}{c}{ Clínicas } \\
\cline { 2 - 5 } Exames & $\mathbf{A}$ & $\mathbf{B}$ & $\mathbf{C}$ & Média \\
& $\mathbf{R} \$$ & $\mathbf{R} \$$ & $\mathbf{R} \$$ & $\mathbf{R} \mathbf{}$ \\
\hline US* & 120,00 & 150,00 & 205,00 & 158,33 \\
TC & 240,00 & 226,00 & 300,00 & 255,33 \\
RM & 500,00 & 570,00 & 620,00 & 563,33 \\
\hline
\end{tabular}

* Valor do exame bilateral de órbitas Legendas:

US - ultra-sonografia
TC - tomografia computadorizada

RM - ressonância magnética

Fontes: Clínicas particulares oftalmológicas e radiológicas na cidade do Rio de Janeiro

cina baseada em evidências (MBE), iniciaram o Projeto Diretrizes. No final de 2002, foram divulgadas cem diretrizes elaboradas por algumas sociedades de especialidades. As doenças da órbita não têm ainda listadas as suas diretrizes ${ }^{(15-16)}$.

\section{Dıscussão}

\section{Honorários médicos}

Entre as principais operadoras de planos de saúde na cidade do Rio de Janeiro pesquisadas, nenhuma 
Tabela 3

Valores dos exames orbitários de US, TC e RM em coeficientes de honorários (CH) e em reais $(R \$)$ de acordo com as tabelas das instituições contratantes de serviços e clínicas particulares na cidade do Rio de Janeiro, 2002/ 2003

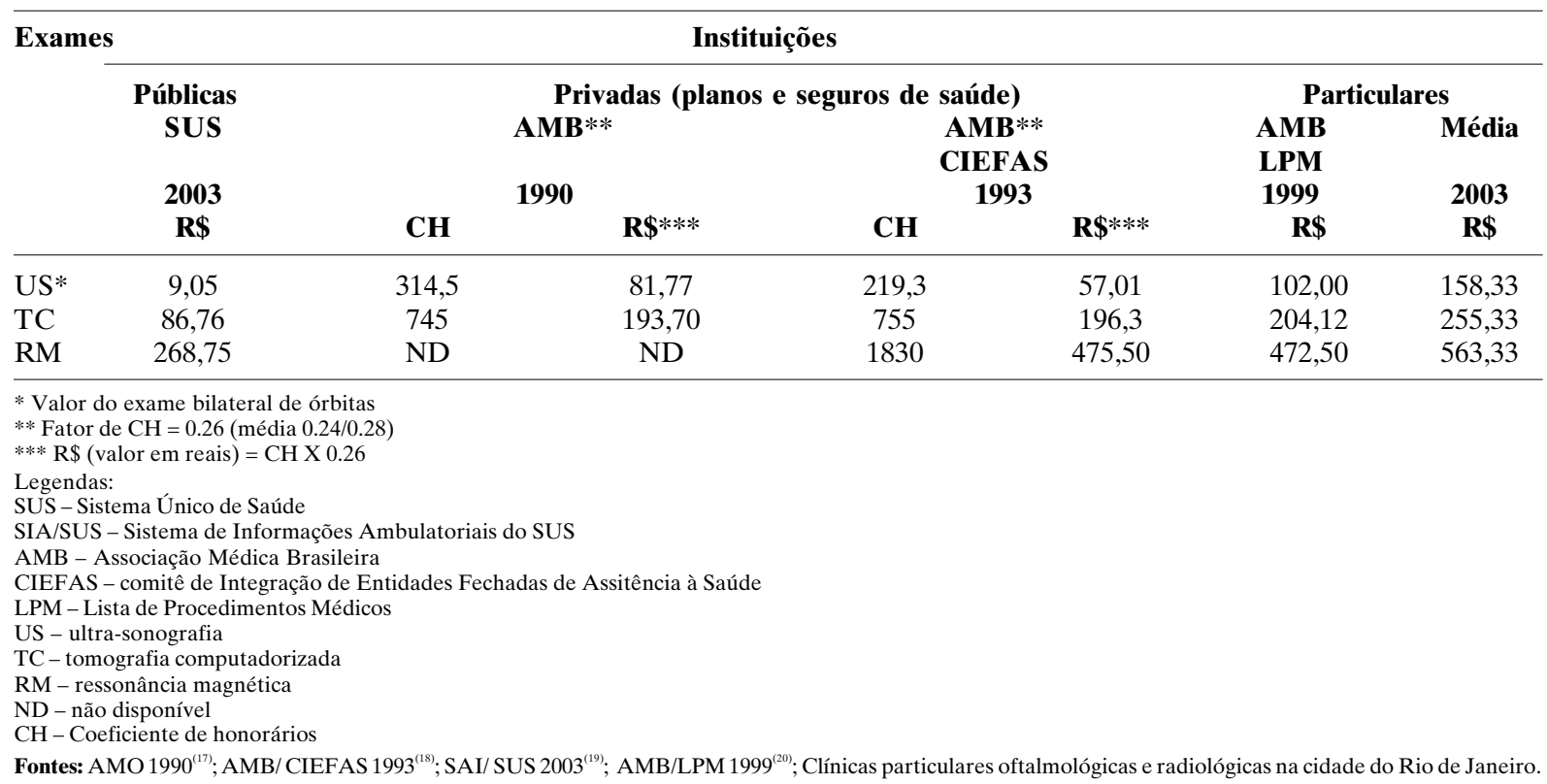

\section{Quadro 2 - Vantagens e desvantagens do exame orbitário de ultra-sonografia}

Vantagens

- Custo baixo ${ }^{(21-22)}$;

- Ausência de radiação ionizante ${ }^{(4,9,21-25)}$;

- Exame relativamente rápido ${ }^{(21,23)}$;

- Pode ser realizado a qualquer momento e quantas vezes for necessário sem risco para o paciente, ideal para o acompanhamento seriado de lesões ou demonstrar a eficácia de um tratamento ${ }^{(4,9,21,25)}$;

- Não exige preparo do paciente, podendo ser realizado em crianças geralmente sem necessidade de sedação ${ }^{(0,23)}$;

- Útil para localizar e delimitar as lesões (melhor no modo B) ${ }^{(9,21,24-26)}$;

- Capacidade de caracterizar os tecidos e medir com precisão o nervo óptico, musculatura extrínseca e massas orbitárias (melhor no modo A padronizado) ${ }^{(4,9,21,24-27)}$;

- Grande importância na avaliação do globo ocular em casos de processos infiltrativos, trauma e na identificação de diversos tipos de corpo estranho ${ }^{(22-23,27-29)}$;

- Geralmente realizado ou supervisionado por oftalmologista $^{(9,22)}$.

\section{Desvantagens}

- Não avalia o ápice orbitário ${ }^{(4,9,21-23,28-29)}$;

- A precisão do exame depende do examinador ${ }^{(21-23,28)}$; adotou a Lista de Procedimentos Médicos (LPM) revisada pela AMB em 1999 (Quadro 1/Tabela 3).

Em relação à US, na tabela CIEFAS 93 está incluído na oftalmologia o procedimento de ultra-sonografia diagnóstica. A tabela AMB 90 utiliza o valor do exame ultra-sonográfico de globo ocular, procedimento da radiologia. Isto explica a redução dos valores de CH 314.5 / R\$ 81,77 - AMB 90 para CH 219.3 / R \$ 57,01 - AMB/ CIEFAS 93 (Tabelas 1 e 3). Atualmente, a LPM 99 foi revisada pela AMB com assessoria da Fundação Instituto de Pesquisas Econômicas (FIPE) ${ }^{(31-32)}$. Nesta nova lista, denominada Classificação Brasileira Hierarquizada de Procedimentos Médicos (CBHPM), os exames foram compatibilizados $^{(33)}$. A RM foi incorporada posteriormente na radiologia e listada na tabela CIEFAS 93, sendo utilizada como referência pelas instituições que adotam a tabela AMB 90 (Tabelas 1 e 3).

Uma pesquisa realizada pelo Conselho Regional de Medicina do Rio de Janeiro (CREMERJ) mostrou que após o reajuste praticado pelo Grupo CIEFAS, em 1997, houve uma defasagem entre janeiro de 1998 a janeiro de 2003 de 88,44\% pelo Índice Geral de Preços do Mercado $(\mathrm{IGP}-\mathrm{M})^{(34)}$. Baseado neste índice, o valor do $\mathrm{CH}$ estaria valendo 0.49 e não 0.26 (média 0.24/0.28 - Quadro 1 ).

Após este levantamento, ocorreram reajustes em julho e agosto de 2004, sendo que algumas operadoras 
passaram a pagar o $\mathrm{CH}$ em torno de $0.30^{(35)}$. Nos serviços radiológicos pesquisados, porém, um alegou que os valores permaneceram os mesmos, em outro houve um reajuste de $7.4 \%$ nos exames de Rx simples e 5\% para US, TC e RM sobre o valor em reais praticados (Tabela 3 ). Dependendo dos acordos individuais com os planos, os contratados admitem fazer descontos de até $20 \%$ destes valores no faturamento.

A desatualização das tabelas e a acentuada defasagem da remuneração, principalmente do SUS (Tabela 3), propiciou uma piora na eficiência dos serviços, levando provedores a reduzir e compensar os custos com um aumento exagerado na quantidade dos exames complementares. Da mesma maneira, os chamados "pacotes" pagos pelo SUS e operadoras, não acompanham os constantes reajustes dos insumos utilizados e outros custos operacionais.

Na tentativa de uma melhoria na situação, foi criada, no Rio de Janeiro, a Central de Convênios, visando ao credenciamento universal dos profissionais e livre escolha para os pacientes de acordo com a sua preferência dos médicos, serviços e clínicas, listados no Livro Regional de Saúde ${ }^{(36-37)}$. Seguindo os mesmos passos, a oftalmologia criou a Cooperativa Estadual de Serviços Administrativos em Oftalmologia (COOESO $)^{(12,38)}$. O ponto fundamental neste processo é o de se estabelecer protocolos na requisição dos exames complementares com guias padronizadas (prevenindo o excesso de exames e glosas) e tabela única com valores mínimos (reembolsáveis) para os procedimentos das especialidades, repudiando a imposição de "pacotes" ou descontos percentuais na remuneração, através da negociação de contratos coletivos. Isto viabilizaria o atendimento inclusive dos pacientes que dependem do SUS e que não podem pagar um plano privado ou os valores de um exame particular (Tabelas 2 e 3). Outro exemplo ocorreu nas cidades de Campos e Barra Mansa (RJ), onde as respectivas prefeituras estão complementando em até duas vezes o valor da tabela SUS, facilitando o atendimento da população nos serviços que se credenciarem, desafogando os serviços públicos já saturados ${ }^{(39)}$.

\section{Vantagens e desvantagens dos exames}

Os três exames avaliados estão presentes em clínicas particulares e conveniadas, públicas e privadas na cidade do Rio de Janeiro. Portanto, é fundamental que o especialista saiba escolher judiciosamente o método mais adequado para o paciente, considerando as informações oferecidas pelo exame e que tenha o conhecimento de suas potencialidades e limitações.

A realização e interpretação da US orbitária depende de poucos ultra-sonografistas experientes e está

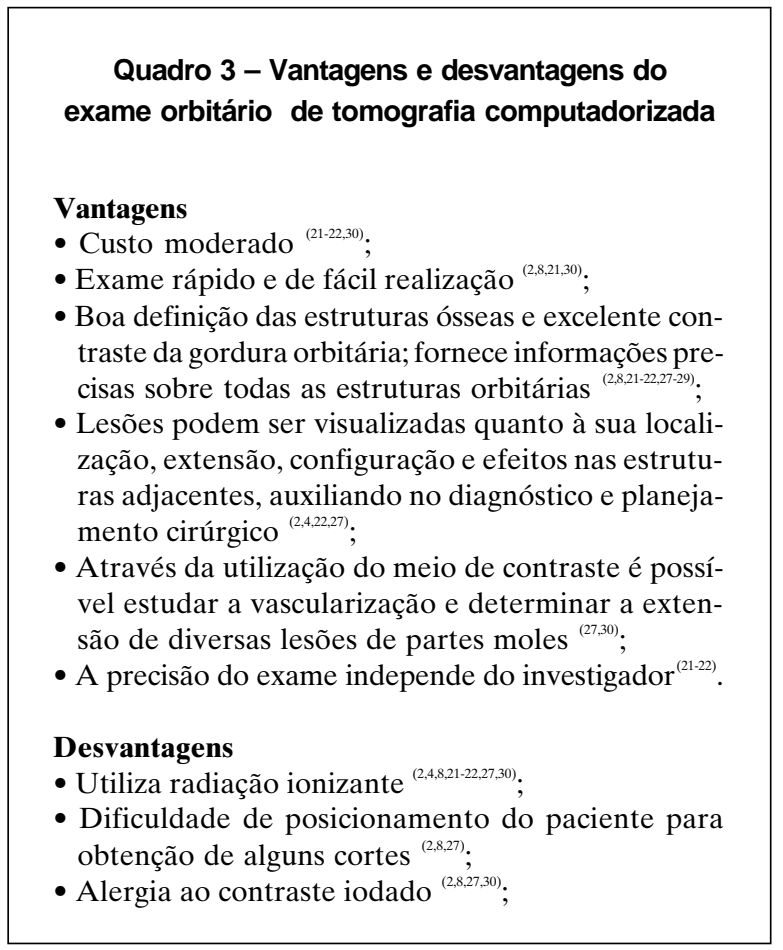

\section{Quadro 4 - Vantagens e desvantagens do exame orbitário de ressonância magnética}

\section{Vantagens}

- Não utiliza radiação ionizante ${ }^{(2,4,21,30)}$;

- Precisão na avaliação e diferenciação entre tecidos moles ${ }^{(2,4,8,21,30)}$;

- Melhor avaliação das vias ópticas ${ }^{(2,8,27)}$;

- Detecção de diversos tipos de corpos estranhos não metálicos ${ }^{(22,28-29)}$;

- Eliminação óssea ${ }^{(27)}$;

- As imagens podem ser obtidas em qualquer plano (multiplanar) ${ }^{(2,8,27,30)}$;

- Possibilita estudo de forma dinâmica com o uso de contraste paramagnético, que praticamente não provoca reações adversas ${ }^{(30)}$;

- A precisão do exame independe do investigador ${ }^{(21-2)}$;

\section{Desvantagens:}

- Custo alto ${ }^{(21-22,30)}$;

- Dificuldade de se detectar calcificações e imprecisão na avaliação de lesões ósseas ${ }^{(2,4,8,21,27)}$;

- Contra-indicado na presença de corpo estranho metálico, marca-passo cardíaco, implantes cocleares, valvas cardíacas $^{(2,22,27.30)}$;

- Tempo de exame longo ${ }^{(2,8,21,30)}$;

- Claustrofobia ${ }^{(8,21,27)}$. 
limitada ao estudo de suas porções anterior e média. É um método de baixo custo, inócuo e tempo de exame relativamente rápido, devendo ser utilizado principalmente no rastreamento de lesões orbitárias suspeitas, podendo indicar ou complementar outros procedimentos (Quadro 2).

Tanto a TCquanto a RM contribuem no diagnóstico da maioria das afecções orbitárias. A TC é o método mais utilizado, apesar dos inconvenientes da radiação ionizante (Quadro 3). A RM, devido ao seu custo alto e demora na aquisição de imagens durante o exame, deve ser requisitada principalmente nos casos mais específicos ou em casos não evidenciados com outros métodos (Quadro4).

\section{Diretrizes clínicas}

Futuras tecnologias de alta resolução, como a TEP, prometem elevar os custos dos exames diagnósticos de imagem, apesar do ganho potencial associado com a sua utilização $^{(5-7)}$. Instituições de saúde de países como Estados Unidos e Inglaterra vêm aumentando seus orçamentos em pesquisas de avaliação tecnológica, principalmente voltadas para análises econômicas de custo-efetividade do impacto resultante em prevenção, diagnóstico e tratamento derivados do uso das tecnologias de imagem, buscando-se evidências confiáveis e de alta qualidade ${ }^{(10-11)}$. Porém, estes estudos demandam tempo e altos custos. Necessitam ser realizados muitas vezes individualmente, para cada indicação clínica, comparando-se novos métodos diagnósticos com outros já consagrados $\mathrm{x}^{(10-11,40)}$. As evidências clínicas e econômicas das tecnologias avaliadas devem responder aos seguintes conceitos:

- Apresenta melhor diagnóstico? (acurácia)

- Funciona em condições ideais? (eficácia)

- Funciona em condições da prática diária? (efetividade)

- Funciona a que custo? (eficiência)

- Qual o valor obtido em função do que se gastou com o uso da tecnologia? (análise custo-efetividade)

Diretrizes e protocolos clínicos relacionados às doenças que acometem a órbita são de fundamental importância, pois a órbita é uma subespecialidade de outras especialidades e envolve exames complementares no diagnóstico. A sua utilização propõe maiores benefícios para os pacientes, contribuindo também na melhoria da eficiência dos serviços e otimização dos recursos. Sendo assim, pesquisas mais detalhadas, em cada uma das doenças em questão, devem ser realizadas para se atingir o custo-efetividade desejado.

\section{Conclusão}

Os exames de US, TC e RM de acordo com a literatura são os mais utilizados e consagrados pela sua efi- cácia no estudo da órbita. Para a melhoria na sua eficiência devemos considerar as seguintes recomendações administrativas:

1- Modernização e atualização das tabelas de honorários com índices mínimos e mais justos na remuneração dos exames com reembolso dos insumos utilizados como filmes radiológicos e contrastes;

2- Promover e estimular a educação médica continuada, evitando o excesso de exames desnecessários ou inapropriados;

3- Elaboração e implementação de diretrizes e protocolos clínicos baseados em evidências nas principais doenças orbitárias que possam ser utilizados como referência na tomada de decisões médicas e administrativas na incorporação e utilização dos referidos procedimentos de imagem.

\section{Abstract}

Purposes: This study focuses the orbital exams of ultrassononography (US), computed tomography (CT) and magnetic resonance imaging (MRI), and has the following purposes: Collecting the paid amounts by Sistema Único de Saúde (SUS), Associação Médica Brasileira $(A M B)$ and private clinic tables. Reviewing the advantages and disadvantages of the referred diagnostic tests. Suggesting administrative efficiency measures related to these tests in the orbit. Methods: The costs were achieved based on the amounts paid by SUS, brazilian medical insurance companies and private clinics in Rio de Janeiro City. Bibliographic research of the advantages and disadvantages of these tests. Results: Amounts of these tests in reais $(R \$)$ : US: 9,05 - SUS / 81,77 - AMB $90 /$ 57,01 - AMB/CIEFAS 93 / 102,00 - AMB/LPM $99 /$ 158,33 - private clinics. CT 86,76-SUS /193,70-AMB90 / 196,30 - AMB/CIEFAS 93 / 204,12 - AMB/LPM 99 / 255,33 - private clinics MRI: 268,75 - SUS / 475,80 AMB 90 and AMB/CIEFAS 93/472,50-AMB/LPM 99 / 563,33 - private clinics. The advantages and disadvantages of these tests were described according to the literature review. Conclusion: Knowing the tests costs and its advantages and disadvantages are important strategies to improve efficiency in diagnostic imaging. It was also concluded the need of new clinical studies that includes economic evaluation of cost-effectiveness in US, $C T$ and MRI exams in orbital diseases which allow the development of clinical practice guidelines or clinical protocols to be applied in medical and administrative decision making.

Keywords: Practice management, medical; Ultrassonography; Tomography, X-ray computed; Magnetic resonance imaging; Orbit 


\section{REFERÊNCIAS}

1. Koch HA, Ribeiro ECO, Tonomura ET, Bahia PRV, Carvalho ACP, Barbalho TMM. Tecnologia e saúde: Uma visão crítica da radiologia. In: Koch HA, Ribeiro EC, Tonomura ET, Bahia PRV, Carvalho ACP, Barbalho TMM, editores. Radiologia na formação do médico geral. Rio de Janeiro: Revinter; 1997. p.1-6.

2. Monteiro MRL. Radiografia, tomografia e ressonância magnética. In: Yamane R, editor. Semiologia ocular. 2a ed. Rio de Janeiro: Cultura Médica; 2003. p.409-29.

3. Dantas AM, Monteiro MRL. Prefácio. In: Dantas AM, Monteiro MLR, editores. Doenças da órbita. Rio de Janeiro: Cultura Médica; 2002.

4. Kincaid MC, Green WR. Diagnostic methods in orbital diseases. Ophthalmology. 1984; 91(6):719-25.

5. Rothschild JM, Khorasani R, Bates DW. Guidelines and decision support help improve image utilization. Diagn Imaging (San Franc). 2000; 22(11):95-7, 99, 101.

6. González Espinosa P, Massardo Vega T, Canessa García J, Humeres Aprá P, Jofré M MJ. Aplicaciones clínicas del PET. Rev Méd Chile. 2002;130(5):569-79.

7. Brasil começa a fazer exame de alta definição. O mundo/ ciência e vida. O Globo. 2002; Dez 2. p.24.

8. Santos AC, Prado PTC, Velasco e Cruz AA. Órbita: II - Imagem. Arq Bras Oftalmol. 1999; 62(2):208-19.

9. Byrne SF, Green RL. The orbit. In: Byrne SF, Green RL, editors. Ultrasound of the eye and orbit. 2nd ed. St. Louis: Mosby; 2002. p. 273.

10. Hutton J. Assessing the cost-effectiveness of imaging technology: recent results, problems and progress in UK. Eur Radiol. 2000; 10 Suppl 3:S427-9.

11. Sullivan DC. NIH and cost-effectiveness studies for imaging. Eur Radiol. 2000; 10 Suppl 3:S408-10.

12. Louzada N. Cooeso propõe relação "ganha x ganha" para garantir direitos de contratados e contratantes. J Bras Oftalmol. 2003; 97:17.

13. Koch HA, Ribeiro ECO, Tonomura ET, Bahia PRV, Carvalho ACP, Barbalho TMM. Apresentação. In: Koch HA, Ribeiro EC, Tonomura ET, Bahia PRV, Carvalho ACP, Barbalho TMM, editores. Radiologia na formação do médico geral. Rio de Janeiro: Revinter; 1997. iv.

14. Yamane R. Exame clínico do bulbo ocular e seus anexos. In: Yamane R, editor. Semiologia ocular. 2a d. Rio de Janeiro: Cultura Médica; 2003. p. 1-7.

15. Projeto Diretrizes. Jornal do Conselho Federal de Medicina 2002;139 (Nov):12- 3.

16. Projeto Diretrizes da Associação Médica Brasileira e Conselho Federal de Medicina [texto na Internet]. [citado $2007 \mathrm{Jul}$ 21]. Disponível em URL: http://www.projetodiretrizes.org.br/

17. Tabela de honorários e serviços da Bradesco Seguros. São Paulo ; Ago/1990.125p.

18. Comunicado CIEFAS n ${ }^{\circ}$ 07/03. Convênio CIEFAS - AMB. Ed. rev. São Paulo: Nov/1993. 74p.

19. Consulta de procedimentos SIA/SUS [citado 2006 Jul 27]. Disponível em URL: http://dtr2001.saude.gov.br/sas/decas/ consultatab1.htm

20. Associação Médica Brasileira. Lista de procedimentos médicos - valores referenciais. Brasília; 1999.172p.

21. Kahaly GJ. Imaging in thyroid-associated orbitopathy. Eur J Endocrinol. 2001; 145(2):107-18.

22. Bocaccio FJL, Domingues CG, Chies MA. Os exames de imagem na avaliação pós-trauma penetrante do globo ocular e da órbita. Rev Bras Oftalmol. 1996; 55(6):21-8.
23. Allemann N. Ultra-sonografia A e B. In: Yamane R, editor. Semiologia ocular. 2a ed. Rio de Janeiro: Cultura Médica 2003. p.337-56.

24. Juaçaba RC, Abreu GB. Histórico e introdução. In: Abreu G, editor. Ultra-sonografia ocular: atlas e texto. $3^{\mathrm{a}}$ ed. rev. ampl. Rio de Janeiro: Cultura Médica; 2002. p.1-2.

25. Lombardi AA, Corrêa ZMS. Ultra-sonografia orbitária. Ecografia normal e técnicas de exame. In: Abreu G, editor. Ultra-sonografia ocular: atlas e texto. $3^{\mathrm{a}}$ ed. rev. amp. Rio de Janeiro: Cultura Médica: 2002. p.231-46.

26. Byrne SF, Green RL. Physics and instrumentation. In: Byrne $\mathrm{SF}$, Green RL, editors. Ultrasound of the eye and orbit. 2nd ed. St. Louis: Mosby; 2002. p.10-1.

27. Dantas AM. Semiologia da órbita. In: Dantas AM, Monteiro MLR, editores. Doenças da órbita. Rio de Janeiro: Cultura Médica; 2002. p.117-37.

28. Casanova FHC, Mello Filho PAA, Nakanami DM, Manso PG. Corpo estranho orgânico intra-orbitário: avaliação tomográfica e conduta. Arq Bras Oftalmol. 2001; 64(4):297301.

29. Monteiro MLR. Traumatismos orbitais. In: Dantas AM, Monteiro MLR, editores. Doenças da órbita. Rio de Janeiro: Cultura Médica; 2002. p.249-72.

30. Batista LP. Como indicar (bem) CT e ressonância [texto na Internet]. [citado 2007 Jul 12]. Disponível em URL: http:// radiology.com.br/rmtc.htm

31. Associação Médica Brasileira. Pela união dos médicos. In: Livro regional de saúde. Listagem ética de médicos, serviços e clínicas. Rio de Janeiro; AMB: 2000. p.19.

32. SOMERJ. Por melhores médicos para uma melhor medicina. In: Livro regional de saúde. Listagem ética de médicos, serviços e clínicas. Rio de Janeiro; 2000. p.24-7.

33. Classificação dos procedimentos médicos está na etapa final Jornal do Cremesp. 2003; 187: 9.

34. Defasagem de $88,44 \%$ pelo IGP-M. Reajuste já. Jornal do Cremerj. 2003; 149: 5 .

35. Negociações com operadoras avançam. Jornal do Cremerj. 2004; 167:7.

36. Central de convênios. Introdução. In: Livro regional de saúde. Listagem ética de médicos, serviços e clínicas. Rio de Janeiro; 2000. p. 7-10.

37. Central de convênios. Apresentação. In: Livro regional de saúde. Listagem ética de médicos, serviços e clínicas. Rio de Janeiro; 2000. p. 3.

38. Louzada N. A hora e a vez das cooperativas médicas de especialidades. Jornal Oftalmológico Jota Zero. 2002; 16(86):36

39. Em Campos, remuneração triplicada. Jornal do Cremerj 2003;148:13.

40. Valk PE. Clinical trials of cost effectiveness in technology evaluation. O J Nucl Med. 2000; 44(2):197-203.
ENDEREÇO PARA CORRESPONDÊNCIA
Abelardo de Souza Couto Júnior
Av. N. S. de Copacabana 1.120 - Gr. 901
CEP 22060-000 - Rio de Janeiro - RJ
Telefax: (21) 2521-0645 / 2287-8346
Email: soluc@ig.com.br 